\title{
Spontaneous Resolution of Massive Fetal Pleural Effusion: A Case Report
}

\author{
Paban Sharma, Harihar Shrestha'
}

Patan Hospital \& MIDAT Hospital ${ }^{\text {? }}$

\begin{abstract}
Isolated fetal pleural effusion is a rare finding in obstetrics. We present a case of fetal pleural effusion during second trimester of the pregnancy which resolved on its own. In the situation of isolated pleural effusion, sometime it may be reasonable to watch and see the progress of the situation.

Key words: Fetal pleural effusion, Ultrasound examination and thoracoabdominal shunting
\end{abstract}

\section{Introduction}

Isolated Pleural effusion in intrauterine life may be one of the finding during routine antenatal ultrasound. Pleural effusion may be the part of generalized fluid collection in the body like in immune and non- immune hydrops or this may be related various syndrome and abnormal baby. If the pleural effusion is isolated and there are no other features of congenital abnormality, prognosis seems to be better. Although the exact cause of isolated fetal effusion is not known, but this may be due to transient fetal infection, brief cardiac failure or transient decreases in fetal colloid osmotic pressure. Therefore, it may be reasonable sometime to make an approach of wait and see, expecting the spontaneous resolution of isolated fetal effusion.

\section{Case}

A 26 yrs lady from Kathmandu, married 2 yrs back as unable to conceive. She had no other significant medical history except bronchial asthma and after treatment, was in the fully controlled state. Her husband was found to be azospermic which however after receiving treatment from subfertility clinic, improved to oligospermia $(<5$ million $/ \mathrm{ml}$ ) All the investigations done for subfertility were found to be normal. She had a normal cycle of 30-37 days with average flow. After few months she conceived spontaneously without having any intervention. Her antenatal check up to 16 weeks was uneventful and three scan done at 8,12 and 16 weeks showed normal findings and corresponding to gestational age. At 20 weeks another ultrasound was done, which showed 20-21 weeks pregnancy with

Correspondence

Paban Sharma.MD

Consultant, Gyne/Obst department, Patan Hospital

paban.Sharma@gmail.com bilateral pleural effusion in the fetus, more on left side with displacement of the heart to the right (Fig 1, 2a/b).

There was no ascites or pericardial effusion. Liquor volume was normal with normal placentation. A second opinion was sought by another sonologist but the findings were similar. Termination of pregnancy was advised looking at the ultrasound finding.

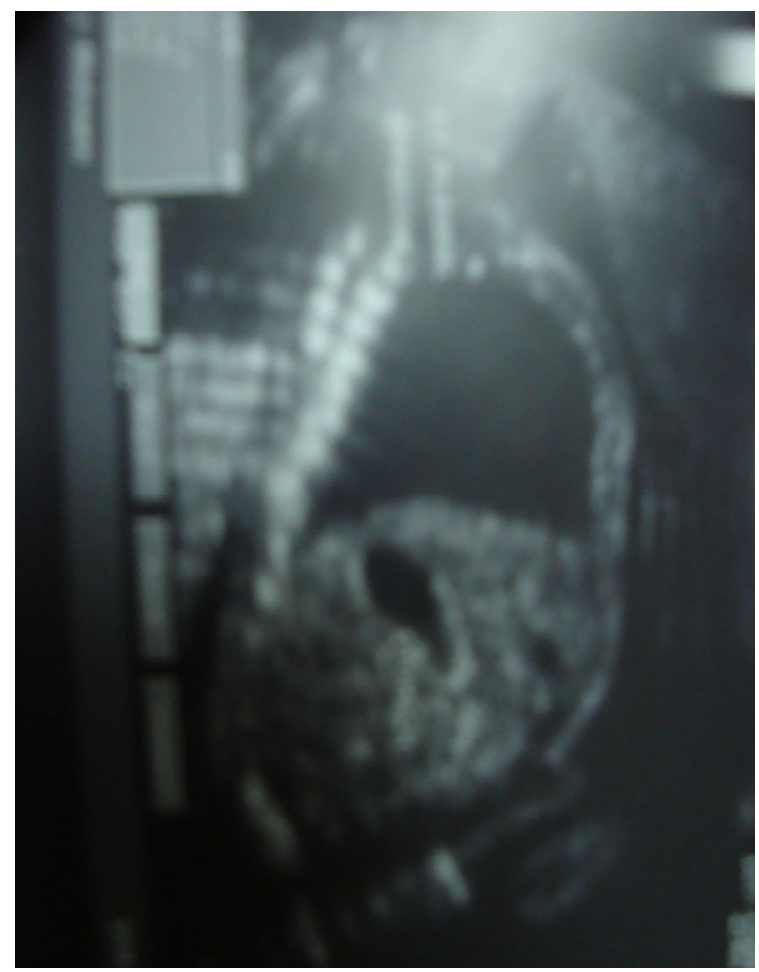

Fig. 1.Fetal pleural effusion 


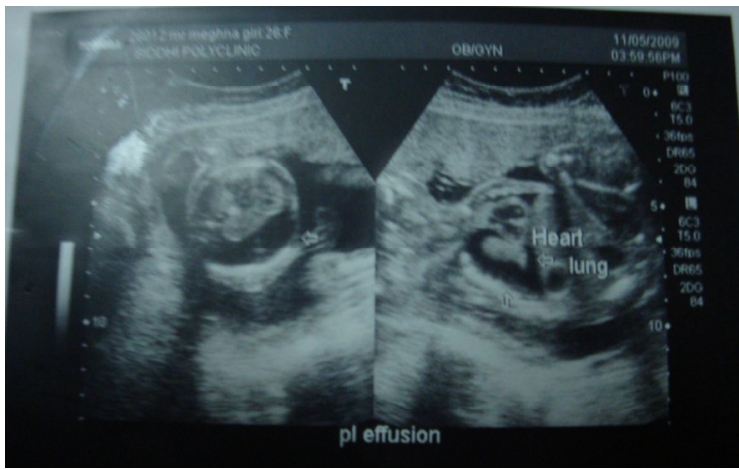

Fig 2a.

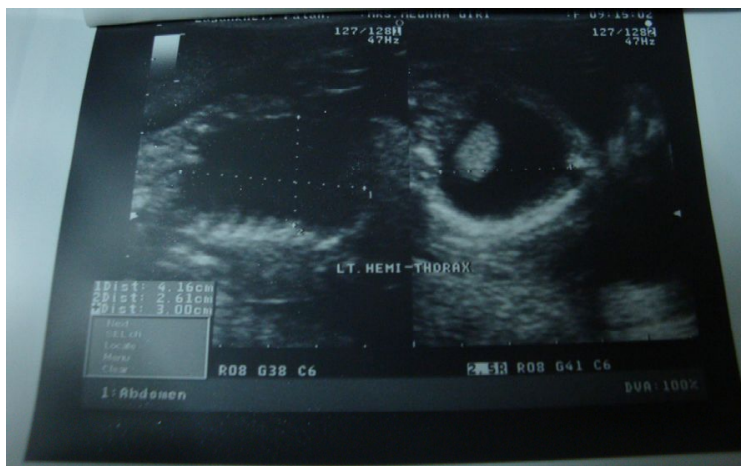

Fig 2b. 2a/b Fetal pleural effusion more on left side

Since this was a pregnancy with oligospermia, they went for another consultation and again ultrasound was done with different sonologist, which also showed similar findings like "The whole of Left hemi-thorax demonstrated pleural effusion, measuring $4.2 \times 2.6 \times 3.0$ $\mathrm{cm}$ with collapsed lung". Small amount of pleural effusion was also seen right. No pericardial effusion and no obvious structural cardiac defect were seen. There was no free fluid in other part of the fetal body. Since this was a very precious pregnancy, they came for another consultation. After discussion with couple few other investigations were done.

Fetal echocardiogram showed no significant structural and functional abnormality of the fetal heart but fetal pleural effusion was noted at that time. TORCH (IgM) screening was negative. Glucose challenge test was $151 \mathrm{mg}$ but $100 \mathrm{gm}$ oral glucose tolerance test was normal. Her blood group was B positive, RPR and HbsAg were negative. Her hemoglobin was normal. Meanwhile literature was reviewed that updated on spontaneous resolution of isolated fetal pleural effusion, so we planned to wait for few more weeks.

Fortunately, subsequent scan after 10 days showed a decrease in pleural effusion and the scan at 24 weeks showed a complete disappearance of pleural effusion. Subsequent antenatal visits were uneventful and ultrasound reports were also normal. After discussion with patient party Elective caesarean delivery was done and a live male baby weighing $2800 \mathrm{gm}$ was delivered.

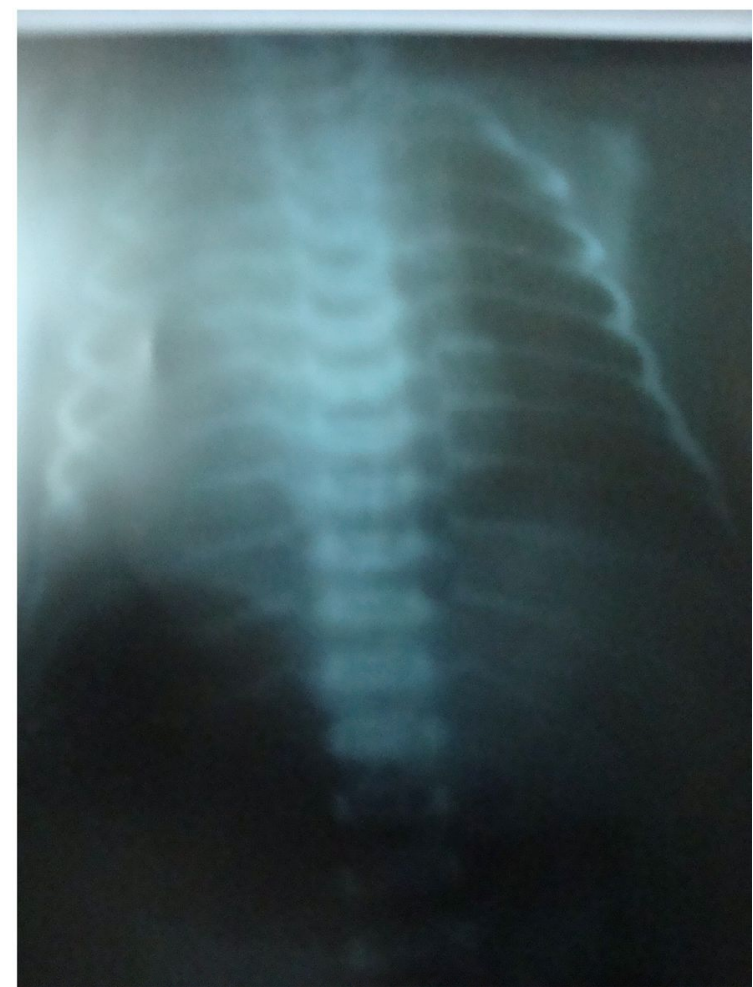

Fig. 3. CXR taken after birth

Baby cried immediately after birth yet the neonate was observed in nursery for few days on account of the history of pleural effusion in fetal life although the Chest $\mathrm{X}$-ray done immediately was normal too. Both mother and baby were discharged on 5th post operative day and followed up in OPD. There was no problem in baby in post natal period.

\section{Comment}

Fetal pleural effusion is accumulation of fluid in the fetal pleural space. The condition is diagnosed on routine ultrasound examination during antenatal check up. Ultrasound of fetal chest will be found to have fluid surrounding the lung on one (unilateral) or both (bilateral) sides. Prenatally, the condition may be an isolated finding (primary pleural effusion) or may occur in association with other conditions (secondary pleural effusion), and may be part of the clinical findings in cases of immune or nonimmune hydrops.

Fetal pleural effusion is a rare condition. The usual prevalence of the condition is 1:10.000 births and male female ratio is 2:1. Most primary congenital effusions are chylous and occur on the right ${ }^{1}$.

Etiology of the condition varies greatly and includes both immune and non-immune hydrops fetalis. The condition is also related to over 50 genetic, Chromosomal (most often trisomy 21 and monosomy $\mathrm{X})$ and sporadic Syndromes ${ }^{1}$. Other associated situations include cystic hygroma, congenital infections such as adenovirus, parvovirus B19 and herpes simplex 
virus type 1 . In a study of 82 cases of fetal pleural effusion (between 1985-1991), etiology of isolated fetal pleural effusion was unknown in most cases. Possible causes included congenital chylothorax, goiter, lung tumors, and infection. Cardiac defects (4.9\%), Down syndrome $(4.9 \%)$, and polydactyly $(1.2 \%)$ were also found to be associated with isolated fetal pleural effusion ${ }^{2}$. Possible etiology of transient pleural effusions may be undetected fetal infection, brief fetal cardiac failure associated with arrhythmias, or transient decreases in fetal colloid osmotic pressure. A fetus with complex pleural effusion is 11.03 times more likely to have a chromosome abnormality than one with an isolated pleural effusion.

The risk of aneuploidy with isolated pleural effusion is $8.6 \%$ and the risk of aneuploidy when pleural effusion is

present in conjunction with other fetal anomalies is $50 \%$. Since the disease process is related to variety of conditions, it needs to be ruled out different possible situation. A detailed ultrasound evaluation of fetal anatomy is mandatory. Fetal echocardiography, fetal Karyotyping, maternal blood count and virology screening including parvovirus B19 and TORCH screening are useful. Looking for maternal blood group and Rh with antibody status and Kleihauer-Betke test of the maternal blood, if needed are the routine investigations in such cases.

The clinical course varies from spontaneous resolution to a progressive increase and the development of hydrops and polyhydramnios due to mediastinal compression. There is a high risk of preterm delivery and in utero death. If severe and long-standing, it has the effect of a space occupying lesion, impeding normal lung development, with the risk of pulmonary hypoplasia and neonatal death1.

Isolated fetal pleural effusions may resolve spontaneously antenatally, or may persist. Spontaneous resolution is not rare and confers a good prognosis. Spontaneous resolution occurred in 11 cases with $100 \%$ survival in a meta-analysis of 124 cases $^{1}$. If spontaneous resolution does not occur, thoracocentesis and drainage is required and sometimes repeated procedures may be necessary if re- accumulation of the fluid occurs. It is essential to rule out the possible structural and chromosomal anomalies to manage the condition. Other factors for the optimal management depends on gestational age, rate of progression, the development of hydrops and associated maternal symptoms. In one study done between 1997 and 2003 shows the survival of fetuses with severe pleural effusions after thoracoamniotic shunting was $48 \% \%^{3}$. Every 2-3 week ultrasound follow up is needed to see the progression of the disease. Pleurodesis have been reported in few cases with variable success ${ }^{4}$.

A number of genetic syndromes and chromosomal abnormalities may be accompanied with pleural effusions, and the risk of recurrence depends on the underlying cause.

\section{Conclusion}

Fetal pleural effusion is a rare condition and optimal management has not been defined so far. Because the natural history of fetal pleural effusion is unknown, observation with serial ultrasound is an alternative to in utero therapy.

\section{References}

2. Maria Angela Rustico, Mariano Lanna, Dario Coviello, John Smoleniec, Umberto Nicolini. Fetal pleural effusion; Prenat Diagn 2007; 27: 793-799.

3. Hagay Z, Reece A, Roberts A, Hobbins JC. Isolated fetal pleural effusion: a prenatal management dilemma. Obstet Gynecol 1993 Jan; 81(1):147-52.

7. Cordero DR, Adra AM, Bonasso PC. Spontaneous resolution of an isolated unilateral fetal pleural effusion: a case report.Spontaneous resolution of an isolated unilateral fetal pleural effusion: a case report. WV Med J. 1996 Jul-Aug; 92(4):194-6.

8. Insertion of pleuro-amniotic shunt for fetal pleural effusion,September 2006, National Institute for Health and Clinical Excellence, MidCity Place, 71 High Holborn, London WC1V 6NA; www.nice.org.uk 